\title{
Adaptive Control for a Class of Nonlinear System with Redistributed Models
}

\author{
Haisen Ke and Jiang Li \\ College of Mechanical and Electrical Engineering, China Jiliang University, Zhejiang, Hangzhou 310018, China \\ Correspondence should be addressed to Haisen Ke, ksea2001@126.com
}

Received 3 November 2011; Accepted 11 April 2012

Academic Editor: Chengyu Cao

Copyright ( $) 2012$ H. Ke and J. Li. This is an open access article distributed under the Creative Commons Attribution License, which permits unrestricted use, distribution, and reproduction in any medium, provided the original work is properly cited.

Multiple model adaptive control has been investigated extensively during the last ten years in which the "switching" or "switching and tuning" have emerged as the mainly approaches. It is the "switching" that can improve the transient performance to some extent and also make it difficult to analyze the stability of the system with multiple models adaptive controller. Towards this goal, this paper develops a novel multiple models adaptive controller for a class of nonlinear system in parameter-strict-feedback form which not only improves the transient performance significantly, but also guarantees the stability of all the states of the closed-loop system. A simulation example is proposed to illustrate the effectiveness of the developed multiple models adaptive controller.

\section{Introductions}

The multiple model adaptive control was introduced to cope with the large parametric uncertainty [1] which always results in large and oscillatory responses or even instable when using the classical adaptive control methods. The multiple models adaptive control [1-7] employing both fixed model and adaptive model have been used to identify the characteristics of the plants, and numerous methods are currently available for controlling such plant satisfactorily. However, the methods mainly focus on the linear time invariant plant $[1,2,4-6]$. The multiple models adaptive controller for nonlinear system is firstly considered in [8], which uses a direct parameter update law to guarantee the stability of the closed-loop system. Then, Ciliz and Cezayirli [9] proposes a different nonlinear multiple models adaptive control which require the condition of persistence of excitation, so that the unknown parameter can be evaluated at the very beginning. Recently, an indirect multiple models adaptive control was developed in [7] which also demonstrated the global asymptotic stability of the closedloop switching system.

As illustrated in the literature that the "switching" (to the closest model) based on the index of performance results in fast response, and tuning (from the closet model) improves the identification and control errors on a slower time scale, which have the assumption that there are abundant models available. Otherwise, the results may be improved less if the number of the identification models is not adequate to achieve the satisfactory response.

In this paper, a novel multiple models adaptive control was considered for the nonlinear system in parameterstrict-feedback form, which retains the advantages of the multiple models adaptive controller, meanwhile facilitate the procedure to analyze and synthesize the controller of the closed-loop system. The approach developed here in which the multiple models adaptive controller are used to play a significantly larger role in the decision making role, results in substantial improvement in performance. Besides, we also reduce the number of the identification models by redistributing the candidate models even as the system is in operation.

\section{Problem Formulation}

Consider the multiple models adaptive control of the following nonlinear parameter-strict-feedback (PSF) system:

$$
\begin{gathered}
\dot{x}_{i}=x_{i+1}+\boldsymbol{\varphi}_{i}^{T}\left(\overline{\mathbf{x}}_{i}\right) \boldsymbol{\theta}, \quad 1 \leq i \leq n-1, \\
\dot{x}_{n}=\beta(\mathbf{x}) u+\boldsymbol{\varphi}_{n}^{T}(\mathbf{x}) \boldsymbol{\theta}, \\
y=x_{1},
\end{gathered}
$$


where $\overline{\mathbf{x}}_{i}=\left[x_{1}, \ldots, x_{i}\right]^{T} \in R^{i}$ and $\mathbf{x} \in R^{n}$ are the state, $u \in R$ is the control input, $\boldsymbol{\theta} \in R^{p}$ is an unknown parameter vector belonging to a known compact set $S$. The functions $\boldsymbol{\varphi}_{i}\left(\overline{\mathbf{x}}_{i}\right)$ and $\beta(\mathbf{x})$ are known smooth functions with $\beta(\mathbf{x}) \neq 0$, for all $\mathbf{x} \in R^{n}$. The focus of this paper is to improve the transient performance in the presence of large parametric uncertainties.

One easly way to improve the transient performance may be choosing sufficiently large high-frequency parameters in the conventional backstepping adaptive control design. Unfortunately, the control efforts can also be very large simultaneously [7]. Alternately, in cope with these difficulties, "switching" or "switching and tuning" have emerged as the leading methods during the last decade.

\section{Multiple Models Adaptive Controller Design}

In order to ensure the stability and transient performance of the system with larger parametric uncertainty, and consequently the boundedness of the state $\mathbf{x}(t)$, the wellestablished results from the classical adaptive control cannot be used directly. Our multiple models adaptive controller contains $N$ parallel operating identification models on which the control law and the adaptive law are based. For improving the transient performance, it is necessary to distribute the initial estimate values of the unknown parameter $\left\{\hat{\boldsymbol{\theta}}_{j}(0)\right\}_{j=1}^{N}$ uniformly in the compact set $S$ to which the unknown parameter $\boldsymbol{\theta}$ belongs. Therefore, at least one $\widehat{\boldsymbol{\theta}}_{j}(0)$ is close to $\boldsymbol{\theta}$, consequently there must exists one or more identification models in its neighborhood. Since adaptive control can perform well when parametric errors are small, it is naturally that the controller developed on the $j$ th identification model can stabilize the system with satisfactorily transient performance.

3.1. Multiple Identification Models. We will run in parallel $N$ identification models with the same structure which take the different initial parameter estimate values $\left\{\hat{\boldsymbol{\theta}}_{j}(0)\right\}_{j=1}^{N}$ uniformly distributed in the compact set $S$ to which the unknown parameter belongs. We first introduce the filters as follows:

$$
\begin{gathered}
\dot{\boldsymbol{\xi}}_{0}=\left(\mathbf{A}_{0}-\lambda \Xi(\mathbf{x}) \Xi^{T}(\mathbf{x}) \mathbf{P}\right)\left(\boldsymbol{\xi}_{0}-\mathbf{x}\right)+f(\mathbf{x}, u), \quad \boldsymbol{\xi}_{0} \in R^{n}, \\
\dot{\boldsymbol{\xi}}=\left(\mathbf{A}_{0}-\lambda \Xi(\mathbf{x}) \Xi^{T}(\mathbf{x}) \mathbf{P}\right) \boldsymbol{\xi}+\Xi(\mathbf{x}), \quad \boldsymbol{\xi} \in R^{n \times p},
\end{gathered}
$$

where

$$
\begin{gathered}
f(\mathbf{x}, u)=\left[\begin{array}{llll}
x_{2} & \ldots & x_{n} & \beta(\mathbf{x}) u
\end{array}\right]^{T}, \\
\Xi(\mathbf{x})=\left[\begin{array}{lll}
\varphi_{1}\left(x_{1}\right) & \ldots & \varphi_{n}(\mathbf{x})
\end{array}\right]^{T} .
\end{gathered}
$$

$\lambda>0$, and $\mathbf{A}_{0}$ is a Hurwitz matrix such that the Lyapunov equation: $\mathbf{P A}_{0}+\mathbf{A}_{0}^{T} \mathbf{P}=-\mathbf{I}$ has a positive definite solution $P$.
Define

$$
\begin{gathered}
\tilde{\mathbf{e}}=\mathbf{x}-\boldsymbol{\xi}_{0}-\boldsymbol{\xi} \boldsymbol{\theta}, \\
\mathbf{e}_{j}=\mathbf{x}-\boldsymbol{\xi}_{0}-\boldsymbol{\xi} \hat{\boldsymbol{\theta}}_{j}, \quad j=1, \ldots, N, \\
\tilde{\boldsymbol{\theta}}_{j}=\boldsymbol{\theta}-\hat{\boldsymbol{\theta}}_{j}, \quad j=1, \ldots, N .
\end{gathered}
$$

It can be derived from (1)-(7) that

$$
\begin{aligned}
\dot{\tilde{\mathbf{e}}} & =\left(\mathbf{A}_{0}-\lambda \Xi(\mathbf{x}) \Xi^{T}(\mathbf{x}) \mathbf{P}\right) \tilde{\mathbf{e}}, \\
\mathbf{e}_{j} & =\xi \tilde{\boldsymbol{\theta}}_{j}+\widetilde{\mathbf{e}}, \quad j=1, \ldots, N .
\end{aligned}
$$

Since $\widetilde{\mathbf{e}}$ converges to zero exponentially, (9) are called identification error equations.

3.2. Controller Design. The controller design involves $N$ models at total and is developed as [10], which can guarantee the asymptotic tracking when there is not identification error and avoid the finite time escape phenomenon when there exists bounded identification error. Now, the first identification model's adaptive controller is given by

$$
u_{1}=\frac{\left[\alpha_{1, n}\left(\mathbf{x}, \hat{\boldsymbol{\theta}}_{1}, y_{r}, \ldots, y_{r}^{n}\right)\right]}{\beta(\mathbf{x})},
$$

where $y_{r}$ is the reference signal to be tracked and $\alpha_{1, n}$ can be recursively designed by

$$
\begin{gathered}
z_{i}=x_{i}-\alpha_{1, i-1}\left(x_{1}, \ldots, x_{i-1}, \widehat{\boldsymbol{\theta}}_{1}, y_{r}, \ldots y_{r}^{i-1}\right), \\
\alpha_{1, i}=-z_{i-1}-c_{1, i} z_{i}-w_{1, i}^{T} \widehat{\boldsymbol{\theta}}_{1}+y_{r}^{i}-s_{1, i} z_{i} \\
+\sum_{k=1}^{i-1}\left(\frac{\partial \alpha_{1, i-1}}{\partial \mathbf{x}_{k}} \mathbf{x}_{k+1}+\frac{\partial \alpha_{1, i-1}}{\partial y_{r}^{k-1}} y_{r}^{k}\right), \\
w_{1, i}\left(x_{1}, \ldots x_{i}, \hat{\boldsymbol{\theta}}_{1}, y_{r}, \ldots, y_{r}^{i-1}\right)=\boldsymbol{\varphi}_{i}-\sum_{k=1}^{i-1} \frac{\partial \alpha_{1, i-1}}{\partial x_{k}} \boldsymbol{\varphi}_{k}, \\
s_{1, i}=k_{1, i}\left|w_{1, i}\right|^{2}+g_{1, i}\left|\frac{\partial \alpha_{1, i-1}}{\partial \hat{\boldsymbol{\theta}}_{1}}\right|^{2},
\end{gathered}
$$

We choose

$$
V_{1}=\frac{1}{2} \sum_{i=1}^{n} z_{i}^{2}
$$


The time derivative of $V_{1}$, computed with (10)-(14), is given by

$$
\begin{aligned}
\dot{V}_{1}= & -\sum_{i=1}^{n} c_{1, i} z_{i}^{2}+\sum_{i=1}^{n}\left(w_{1, i}^{T} \tilde{\boldsymbol{\theta}}_{1}-\frac{\partial \alpha_{1, i-1}}{\partial \hat{\boldsymbol{\theta}}_{1}} \dot{\hat{\boldsymbol{\theta}}}_{1}\right) z_{i} \\
& -\sum_{i=1}^{n}\left(k_{1, i}\left|w_{1, i}\right|^{2}+g_{1, i}\left|\frac{\partial \alpha_{1, i-1}}{\partial \hat{\boldsymbol{\theta}}_{1}}\right|^{2}\right) z_{i}^{2} \\
\leq & -\sum_{i=1}^{n} c_{1, i} z_{i}^{2}-\sum_{i=1}^{n} k_{1, i}\left|w_{1, i} z_{i}-\frac{1}{2 k_{1, i}} \tilde{\boldsymbol{\theta}}_{1}\right|^{2} \\
& -\sum_{i=1}^{n} g_{1, i}\left|\frac{\partial \alpha_{1, i-1}}{\partial \hat{\boldsymbol{\theta}}_{1}} z_{i}-\frac{1}{2 g_{1, i}} \dot{\hat{\boldsymbol{\theta}}}_{1}\right|^{2} \\
& +\sum_{i=1}^{n} \frac{1}{4 k_{1, i}}\left|\tilde{\boldsymbol{\theta}}_{1}\right|^{2}+\sum_{i=1}^{n} \frac{1}{4 g_{1, i}}\left|\dot{\hat{\boldsymbol{\theta}}}_{1}\right|^{2} \\
\leq & -\sum_{i=1}^{n} c_{1, i} z_{i}^{2}+\sum_{i=1}^{n} \frac{1}{4 k_{1, i}}\left|\tilde{\boldsymbol{\theta}}_{1}\right|^{2}+\sum_{i=1}^{n} \frac{1}{4 g_{1, i}}\left|\dot{\hat{\boldsymbol{\theta}}}_{1}\right|^{2},
\end{aligned}
$$

with $c_{1, i}, k_{1, i}, g_{1, i}$ being designed parameters. Equation (16) implies the boundedness of the states of $z_{i}, 1 \leq i \leq n$, and which in turn indicates the boundedness of the states of $x_{i}, 1 \leq i \leq n$ and control $u_{1}$ on the conditions of $\widetilde{\boldsymbol{\theta}}$ and $\dot{\hat{\boldsymbol{\theta}}}$ are bounded which will be proved later. The rest of $N-1$ controllers can be designed and analyzed similarly which can also guarantee the boundedness of the states of $z_{i}, 1 \leq i \leq n$, and which in turn indicates the boundedness of the states of $x_{i}, 1 \leq i \leq n$ and control $u_{j}, j=1, \ldots, N$.

3.3. Construction of Equivalent Control. In this section, the crucial point is that the transient performance can be improved significantly, and at the same time the switching between the identification models can be avoided. Besides, the information provided by all the identification models is to be utilized efficiently. For the complement of the goals mentioned, instead of using the estimate values of the model with the minimum of performance criterion to reinitial an adaptive controller, a convex combination of all the $N$ models is used to generate the control of the plant as

$$
u=\sum_{j=1}^{N} \gamma_{j} u_{j}
$$

and the adaptive update law as

$$
\dot{\hat{\boldsymbol{\theta}}}_{j}=\boldsymbol{\Gamma} \frac{\boldsymbol{\xi}^{T} \mathbf{e}_{j}}{1+v|\boldsymbol{\xi}|^{2}}, \quad \boldsymbol{\Gamma}=\boldsymbol{\Gamma}^{T}>0, v>0, j=1, \ldots, N,
$$

where $\gamma_{j}$ are nonnegative values satisfying $\sum_{j=1}^{N} \gamma_{j}=1$, and $\gamma_{j}$ can be calculated from

$$
\gamma_{j}=\frac{\left(1 / J_{j}\right)}{\sum_{j=1}^{N}\left(1 / J_{j}\right)},
$$

where $J_{j}$ is the performance indices of the form:

$$
J_{j}(t)=\alpha e_{j}^{2}(t)+\beta \int_{t_{0}}^{t} e_{j}^{2}(\tau) d \tau, \quad a \geq 0, \beta>0,
$$

with $t_{0}$ can be reset when the identification models is redistributed.
3.4. Redistribution of the Identification Models. In this section, the goal is that the transient performance can be improved significantly as far smaller numbers of the identification models as possible. As is illustrated in the literature, the classical adaptive control can cope with the control of linear time invariant system with unknown parameters and achieve satisfactory closed-loop objective only if the plant parametric uncertainty is small. So if the number of the identification models that can be used is abundantly large, the "switching" or "switching and tuning" scheme may act on satisfactorily. Otherwise, the multiple models adaptive control cannot work as expected when the numbers of identification models available is relatively smaller compared with the size of the uncertainty region. Inspired by the "switching" techniques [11-13], we consider the method in which the location of the identification models can be redistributed. From (8) and (9), it can be concluded that the $\widetilde{\mathbf{e}}=0$ can be achieved by choosing the initial values of $\xi_{0}$ and $\xi$ as long as the initial state $x_{0}$ is known or there exists $T>0$ such that

$$
\mathbf{e}_{j}=\boldsymbol{\xi} \widetilde{\boldsymbol{\theta}}_{j}, \quad j=1, \ldots, N, t>T .
$$

It is obviously that the errors $\mathbf{e}_{j}$ and $\tilde{\boldsymbol{\theta}}_{j}, j=1, \ldots, N$ are linearly related. This implies that the index of the performance $J_{j}(t)$ is a quadratic function of the unknown parameter vector $\tilde{\boldsymbol{\theta}}_{j}$. Since $\xi^{T} \xi$ is not negative definite, it follows that the performance indices of all the models are merely points on a time-varying quadratic surface, whose minimum corresponds to the plant indicating the mostly closet identification model $M_{j}$ (corresponds to the parameter $\left.\tilde{\boldsymbol{\theta}}_{j}\right)$. So we can redistribute the other $(N-1)$ models $M_{k}(k \neq j)$ as

$$
\overline{\boldsymbol{\theta}}_{k}=\frac{\sqrt{J_{k}}}{\sqrt{J_{k}}+\sqrt{J_{j}}} \boldsymbol{\theta}_{j}+\frac{\sqrt{J_{j}}}{\sqrt{J_{k}}+\sqrt{J_{j}}} \boldsymbol{\theta}_{k} .
$$

By introducing the minimum of interval time $T_{\min }$ into our switching scheme to ensure a finite number of switching.

\section{Stability Analysis}

Theorem 1. Suppose the multiple models adaptive controller (17) and adaptive law (18) presented in this paper is applied to system (1). Then, for all initial conditions, all closed-loop states are bounded on $[0, \infty)$, and asymptotic tracking can be achieved, that is, $\operatorname{Lim}_{t \rightarrow \infty} z(t)=0$ or $y(t)=y_{r}(t)$ as $t \rightarrow \infty$.

Proof. Since all $N$ models are identical structure and only with different initial estimate parameters, it follows that each controller acts on the system is only different from each other at the weight (each of the controllers can be designed with the same structure and designed parameters).

When we choose the whole candidate Lyapunov function as

$$
V=\frac{1}{2} \sum_{i=1}^{n} \mathrm{z}_{i}^{2}
$$


It is obvious that (23) can be divided into

$$
V=\frac{1}{2} \sum_{i=1}^{n} \mathrm{z}_{i}^{2}=\sum_{j=1}^{N} \gamma_{\mathrm{j}} \frac{1}{2} \sum_{i=1}^{n} \mathrm{z}_{i}^{2}=\sum_{j=1}^{N} \gamma_{\mathrm{j}} V_{j} .
$$

As illustrated by (16), each of the controllers can guarantee the boundedness of the states of $z_{i}, 1 \leq i \leq n$ at its portion, which accompanied with the control (17), and weighting coefficient (19) can establish the boundedness of the states of $z_{i}, 1 \leq i \leq n$.

Next, we prove the controller (17) and adaptive law (18) can also guarantee the asymptotic tracking of the closed-loop system states. It can be computed from (3) that

$$
\begin{aligned}
\frac{d}{d t}\left(\xi \mathbf{P} \xi^{T}\right) & =-\boldsymbol{\xi} \xi^{T}-2 \lambda \xi \mathbf{P} \Xi^{T} \Xi \mathbf{P} \boldsymbol{\xi}^{T}+\xi \mathbf{P} \Xi^{T}+\Xi \mathbf{P} \xi^{T} \\
& =-\xi \xi^{T}-2 \lambda\left(\Xi \mathbf{P} \xi^{T}-\frac{1}{2 \lambda} \mathbf{I}\right)^{T}\left(\Xi \mathbf{P} \xi^{T}-\frac{1}{2 \lambda} \mathbf{I}\right)+\frac{1}{2 \lambda},
\end{aligned}
$$

which shows $\xi$ is bounded regardless of the state $\mathbf{x}$. Let $\mathrm{V}_{j}=$ $\left(\tilde{\boldsymbol{\theta}}_{j}^{T} \boldsymbol{\Gamma}^{-1} \tilde{\boldsymbol{\theta}}_{j}+\widetilde{\mathbf{e}}^{T} \widetilde{\mathbf{e}}\right) / 2$, it can be derived that

$$
\begin{aligned}
\dot{\mathrm{V}}_{j} & =-\frac{\mathbf{e}_{j}^{T}\left(\mathbf{e}_{j}-\tilde{\mathbf{e}}\right)}{1+v|\boldsymbol{\xi}|^{2}}+\widetilde{\mathbf{e}}^{T}\left(\mathbf{A}_{0}-\lambda \Xi(\mathbf{x}) \Xi^{T}(\mathbf{x}) \mathbf{P}\right) \tilde{\mathbf{e}} \\
& \leq-\frac{3}{4} \frac{\mathbf{e}_{j}^{T} \mathbf{e}_{j}}{1+v|\boldsymbol{\xi}|^{2}},
\end{aligned}
$$

without loss of generality, we can design the parameter satisfies $\mathbf{A}_{0}-\lambda \Xi(\mathbf{x}) \Xi^{T}(\mathbf{x}) \mathbf{P}>I, I$ is a unit matrix. Therefore, $\tilde{\mathbf{e}}, \widetilde{\boldsymbol{\theta}}_{j}, j=1, \ldots, N$ are all bounded, which companied with the boundedness of $\boldsymbol{\xi}$, further yields from $\mathbf{e}_{j}=\boldsymbol{\xi} \widetilde{\boldsymbol{\theta}}_{j}+\widetilde{\mathbf{e}}$ that $\mathbf{e}_{j}$ is bounded. It can be also concluded from (26) that $\mathbf{e}_{j}$ is squarely integrable on $[0, \infty)$. Furthermore, we can also conclude from (18) that $\dot{\hat{\boldsymbol{\theta}}}_{j}$ is bounded, which can accomplish the assumption that it is bounded. We can now give the asymptotically tracking control analysis.

The time derivate of identification error is given by

$$
\dot{\mathbf{e}}_{j}=\left(\mathbf{A}_{0}-\lambda \Xi \Xi^{T} \mathbf{P}\right) \mathbf{e}_{j}+\Xi \tilde{\boldsymbol{\theta}}_{j}-\boldsymbol{\xi} \dot{\hat{\boldsymbol{\theta}}}_{j} .
$$

Due to the boundedness of all the closed-loop system states $\dot{\mathbf{e}}_{j}, \dot{\mathbf{e}}_{j}, j=1, \ldots, N$ are also bounded, so by Barbalat's lemma, we must have $\operatorname{Lim}_{t \rightarrow \infty} \mathbf{e}_{j}(t)=0$ and since $\operatorname{Lim}_{t \rightarrow \infty} \int_{t_{1}}^{t} \dot{\mathbf{e}}_{j}(\tau) d \tau=\lim _{t \rightarrow \infty} \mathbf{e}_{j}(t)-\mathbf{e}_{j}\left(t_{1}\right)<\infty$, we further have $\operatorname{Lim}_{t \rightarrow \infty} \dot{\mathbf{e}}_{j}(t)=0$. Then, it can be concluded from (18) that $\operatorname{Lim}_{t \rightarrow \infty} \dot{\hat{\boldsymbol{\theta}}}_{j}=0$ which accompanied with (27) implies $\operatorname{Lim}_{t \rightarrow \infty} \Xi \tilde{\boldsymbol{\theta}}_{j}=0$ and in turn leads to

$$
\operatorname{Lim}_{t \rightarrow \infty} N_{j}\left(z, \hat{\boldsymbol{\theta}}, y_{r}\right) \Xi \tilde{\boldsymbol{\theta}}_{j}=0,
$$

where

$$
N_{j}\left(z, \hat{\boldsymbol{\theta}}_{j}, y_{r}\right)=\left[\begin{array}{cccc}
1 & 0 & & 0 \\
-\frac{\partial \alpha_{j, 1}}{\partial x_{1}} & 1 & 0 & \\
\vdots & & \ddots & 0 \\
-\frac{\partial \alpha_{j, n-1}}{\partial x_{1}} & -\frac{\partial \alpha_{j, n-1}}{\partial x_{2}} & & 1
\end{array}\right] .
$$

By direct calculating, the differentiation of $z$ with parametric controller $u_{j}$ can be described, in $z$ coordination, by

$$
\begin{aligned}
\dot{\mathrm{z}}= & -\sum_{i=1}^{n} c_{j, i} z_{i}+N_{j}\left(z, \hat{\boldsymbol{\theta}}, y_{r}\right) \Xi \tilde{\boldsymbol{\theta}}_{j}-\frac{\partial \alpha_{j, i-1}}{\partial \hat{\boldsymbol{\theta}}_{j}} \dot{\hat{\boldsymbol{\theta}}}_{j} \\
& -\sum_{i=1}^{n}\left(k_{j, i}\left|w_{j, i}\right|^{2}+g_{j, i}\left|\frac{\partial \alpha_{j, i-1}}{\partial \hat{\boldsymbol{\theta}}_{j}}\right|^{2}\right) z_{i} .
\end{aligned}
$$

From (28), accompanied with $\operatorname{Lim}_{t \rightarrow \infty} \dot{\hat{\boldsymbol{\theta}}}_{j}=0$ and the designed parameters are all positive, it can be easily concluded that $\operatorname{Lim}_{t \rightarrow \infty} z(t)=0$, and thus $\operatorname{Lim}_{t \rightarrow \infty} \mathbf{z}_{1}(t)=$ $\operatorname{Lim}_{t \rightarrow \infty}\left(y(t)-y_{r}(t)\right)=0$. The proof is completed.

\section{Simulation}

Consider the following second-order nonlinear system:

$$
\begin{gathered}
\dot{x}_{1}=x_{2}+\theta_{1} x_{1}+\theta_{2} x_{1}^{2}, \\
\dot{x}_{2}=u, \\
y(t)=x_{1}(t),
\end{gathered}
$$

where $\theta_{1} \in[1,5]$ and $\theta_{2} \in[1,40]$ are unknown parameters. The output $y(t)=x_{1}(t)$ is to asymptotically track the reference signal $y_{r}(t)=\sin 2 t$.

In simulation, the parametric controller is developed as (10)-(14) and (17)-(19) with $v=0, \Gamma=5, c_{j, 1}=c_{j, 2}=4$, $k_{j, 1}=k_{j, 2}=g_{j, 2}=0.1, j=1, \ldots, N, \alpha=\beta=1, T_{\min }$ is 5 units of time. Since in (31), the unknown parameter appears only in the first equation, the filter can be constructed as [1] to reduce filter dynamic order:

$$
\begin{gathered}
\dot{\boldsymbol{\xi}}_{0}=-c\left(\boldsymbol{\xi}_{0}-\mathbf{x}\right)+x_{2}, \quad \boldsymbol{\xi}_{0} \in R^{1}, \\
\dot{\boldsymbol{\xi}}=-c \boldsymbol{\xi}+\left[x_{1}, x_{1}^{2}\right], \quad \boldsymbol{\xi} \in R^{1 \times 2},
\end{gathered}
$$

where $c=10$.

The unknown parameter is $\left[\theta_{1}, \theta_{2}\right]=[4.4,38.5]$; the number of the multiple identification models is $N=4$; for convenience to comparison with [7], the initial plant state is $\left[x_{1}(0), x_{2}(0)\right]=[0.5,-10]$; the same initial filter states are $\xi_{0}=0.5, \xi=\left[\begin{array}{ll}0 & 0\end{array}\right]$, and the initial estimate parameters for model 1 , model 2 , model 3 , and model 4 are $\hat{\boldsymbol{\theta}}_{1}(0)=$ $[1,1]^{T}, \hat{\boldsymbol{\theta}}_{2}(0)=[1,5]^{T}, \hat{\boldsymbol{\theta}}_{3}(0)=[5,1]^{T}, \hat{\boldsymbol{\theta}}_{4}(0)=[5,40]^{T}$, respectively. Figures $1-4$ depict the simulation results.

These simulation results clearly showed that the multiple models adaptive controller presented in this paper guarantees the boundedness of all the states in the closed-loop system and achieves the asymptotic tracking of the output.

Figure 1 is the output $y(t)$, which demonstrates that the multiple models adaptive controller developed in this paper has the similar property as shown in [7] and is significantly better than using the classical adaptive control. Figures 2 and 3 are the control inputs which show that the multiple model adaptive control can reduce the maximum control input dramatically. Besides, it seems to conclude that the multiple model adaptive control proposed in this paper has the similar property and so the trajectory is nearly to overlap 


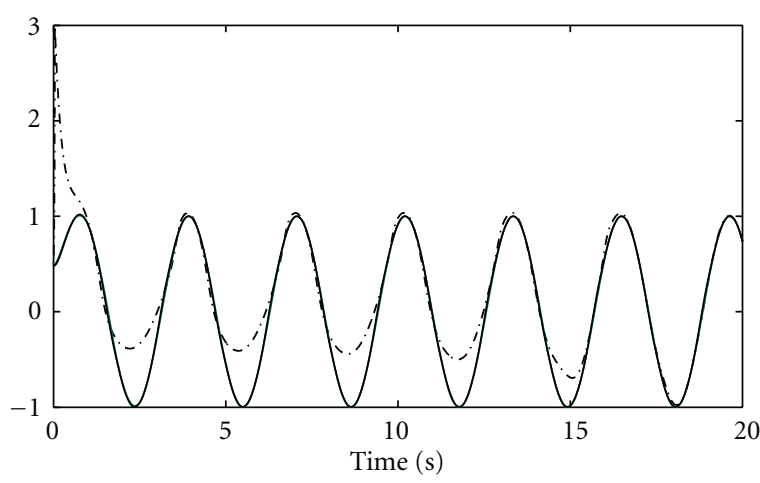

Figure 1: Output $y(t)$. dash-dotted line for the classical adaptive control, dashed line for the multiple model case $(N=200)$ as in [7], and solid line for the multiple identification model developed in this paper.

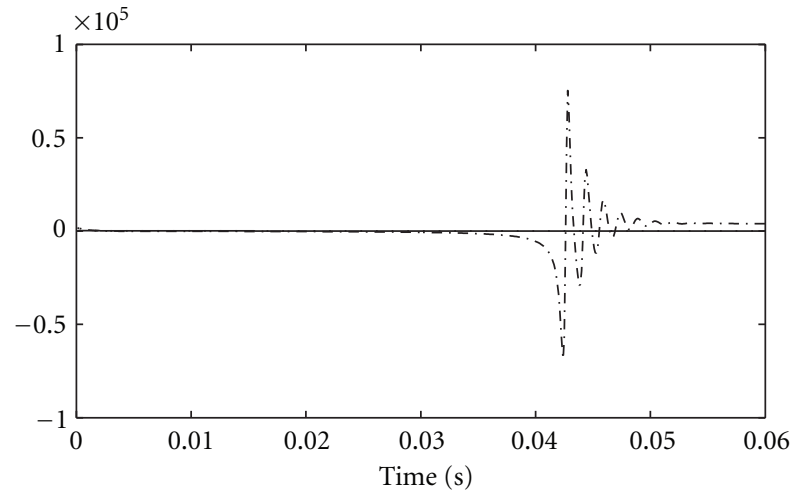

FIGURE 2: Control $u(t)$ on the time interval [0, 0.06]. dash-dotted line for the classical adaptive control, dashed line for the multiple model case $(N=200)$ as in [7], and solid line for the multiple identification model developed in this paper.

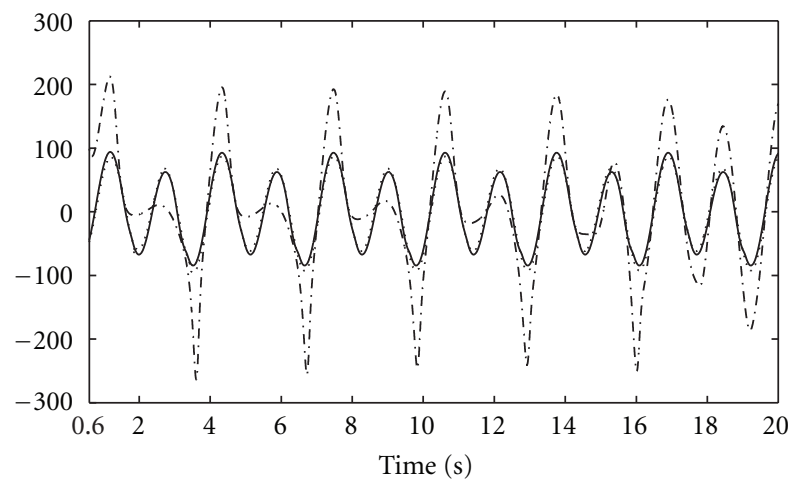

FIGURE 3: Control $u(t)$ on the time interval $[0.6,20]$. dash-dotted line for the classical adaptive control, dashed line for the multiple model case $(N=200)$ as in [7], and solid line for the multiple identification model in this paper.

from Figures 1-3 because the method in [7] uses more identification models than ours. Figure 4 is the trajectory of the redistribution of the identification models which can find the most suitable identification model and enhance the transient performance.

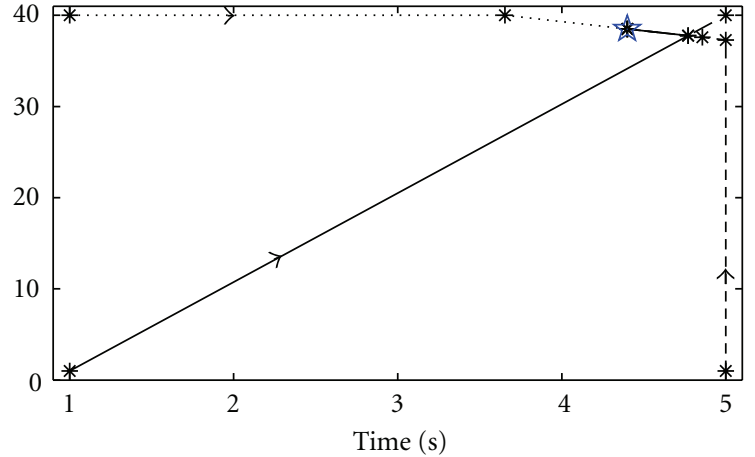

FIGURE 4: The redistribution trajectory of the identification models.

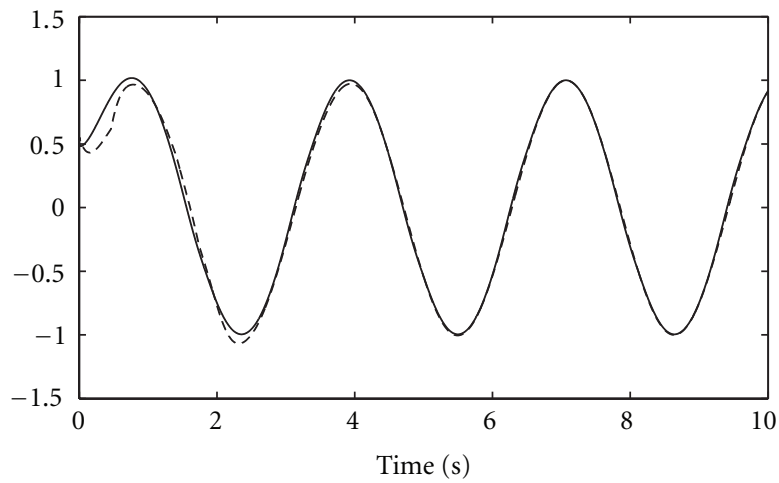

Figure 5: Output $y(t)$. dashed line for the multiple model case $(N=4)$ as in [7], solid line for the multiple identification model developed in this paper.

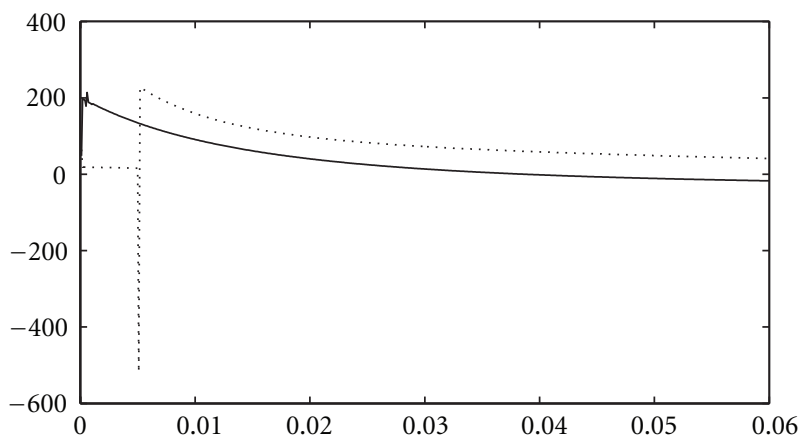

FIgure 6: Control $u(t)$ on the time interval [0, 0.06]. dashed line for the multiple model case $(N=4)$ in [7], and solid line for the multiple model developed in this paper.

Next, we can compare the approach presented in this paper with the method developed in [7] with the multiple identification models $(N=200)$ is set to $(N=4)$, which is the same identification models used in our approach. Figures 5-7 depict the simulation results.

Figure 5 is the output $y(t)$ with the multiple models adaptive controller, which shows the approach developed in this paper is superior to the method presented in [7]. Figures 6 and 7 are the control inputs which show that the multiple model adaptive control developed in this paper has 


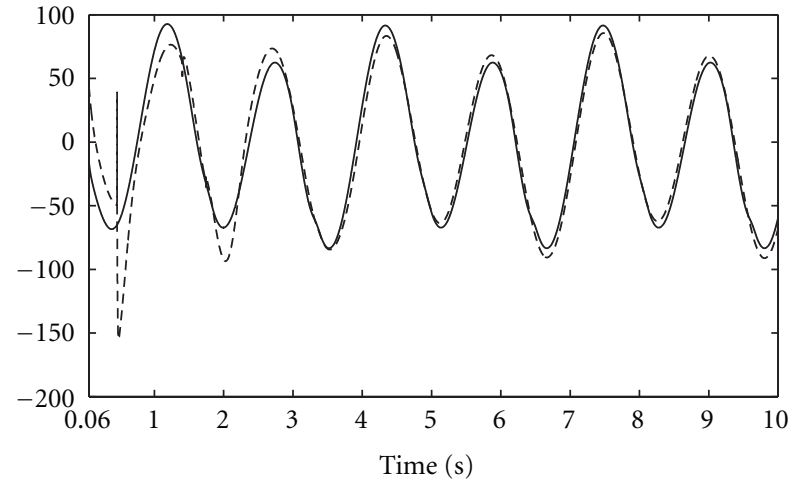

Figure 7: Control $u(t)$ on the time interval $[0.06,10]$. dashed line for the multiple model case $(N=4)$ in [7], and solid line for the multiple model developed in this paper.

better properties than the method presented in [7] which has switching and larger control input.

\section{Conclusions}

In this paper, a novel multiple models adaptive controller was developed for a class of nonlinear systems. The multiple models technique was used to describe the most appropriate model at different environments. If the number of the identification models that can be used is abundantly large, the "switching" or "switching and tuning" scheme may act on satisfactorily. Otherwise, the multiple models adaptive control cannot work as expected when the number of identification models available is relatively small compared with the size of the uncertainty region. So we consider the method in which the location of the identification models can be redistributed. Unlike previous results, we do not require a switching scheme to guarantee the most appropriate model to be switched into the controller design which can simplify the analysis of the stability of the closed-loop system.

\section{Acknowledgments}

The authors would like to thank the reviewers and the editors for their helpful and insightful comments for further improvement of the quality of this work. The work is supported by the National Natural Science Foundation of China (nos. 60674023 and 60905034) and the Zhejiang Provincial Natural Science Foundation of China (no. Y12F030119).

\section{References}

[1] K. S. Narendra and J. Balakrishnan, "Improving transient response of adaptive control systems using multiple models and switching," IEEE Transactions on Automatic Control, vol. 39, no. 9, pp. 1861-1866, 1994.

[2] K. S. Narendra and J. Balakrishnan, "Adaptive control using multiple models," IEEE Transactions on Automatic Control, vol. 42, no. 2, pp. 171-187, 1997.

[3] J. P. Hespanha, D. Liberzon, and A. S. Morse, "Overcoming the limitations of adaptive control by means of logic-based switching," Systems and Control Letters, vol. 49, no. 1, pp. 4965, 2003.

[4] Z. Han and K. S. Narendra, "Multiple adaptive models for control," in 2010 49th IEEE Conference on Decision and Control, CDC 2010, pp. 60-65, usa, December 2010.

[5] K. S. Narendra and Z. Han, "The changing face of adaptive control: the use of multiple models," Annual Reviews in Control, vol. 35, no. 1, pp. 1-12, 2011.

[6] K. S. Narendra and K. George, "Adaptive control of simple nonlinear systems using multiple models," in 2002 American Control Conference, pp. 1779-1784, usa, May 2002.

[7] X. D. Ye, "Nonlinear adaptive control using multiple identification models," Systems and Control Letters, vol. 57, no. 7, pp. 578-584, 2008.

[8] K. S. Narendra and K. George, "Adaptive control of simple nonlinear systems using multiple models," in 2002 American Control Conference, pp. 1779-1784, usa, May 2002.

[9] M. K. Ciliz and A. Cezayirli, "Increased transient performance for the adaptive control of feedback linearizable systems using multiple models," International Journal of Control, vol. 79, no. 10, pp. 1205-1215, 2006.

[10] M. Krstic and P. V. Kokotovic, "Adaptive nonlinear design with controller-identifier separation and swapping," IEEE Transactions on Automatic Control, vol. 40, no. 3, pp. 426-440, 1995.

[11] H. S. Ke and X. D. Ye, "Robust adaptive controller design for a class of nonlinear systems with unknown high frequency gains," Journal of Zhejiang University, vol. 7, no. 3, pp. 315320, 2006.

[12] P. Bashivan and A. Fatehi, "Improved switching for multiple model adaptive controller in noisy environment," Journal of Process Control, vol. 22, no. 2, pp. 390-396, 2012.

[13] H. H. Xiong and S. Y. Li, "Satisfying optimal control of switching multiple models based on mixed logic dynamics," International Journal of Modelling, Identification and Control, vol. 10, no. 1-2, pp. 175-180, 2010. 

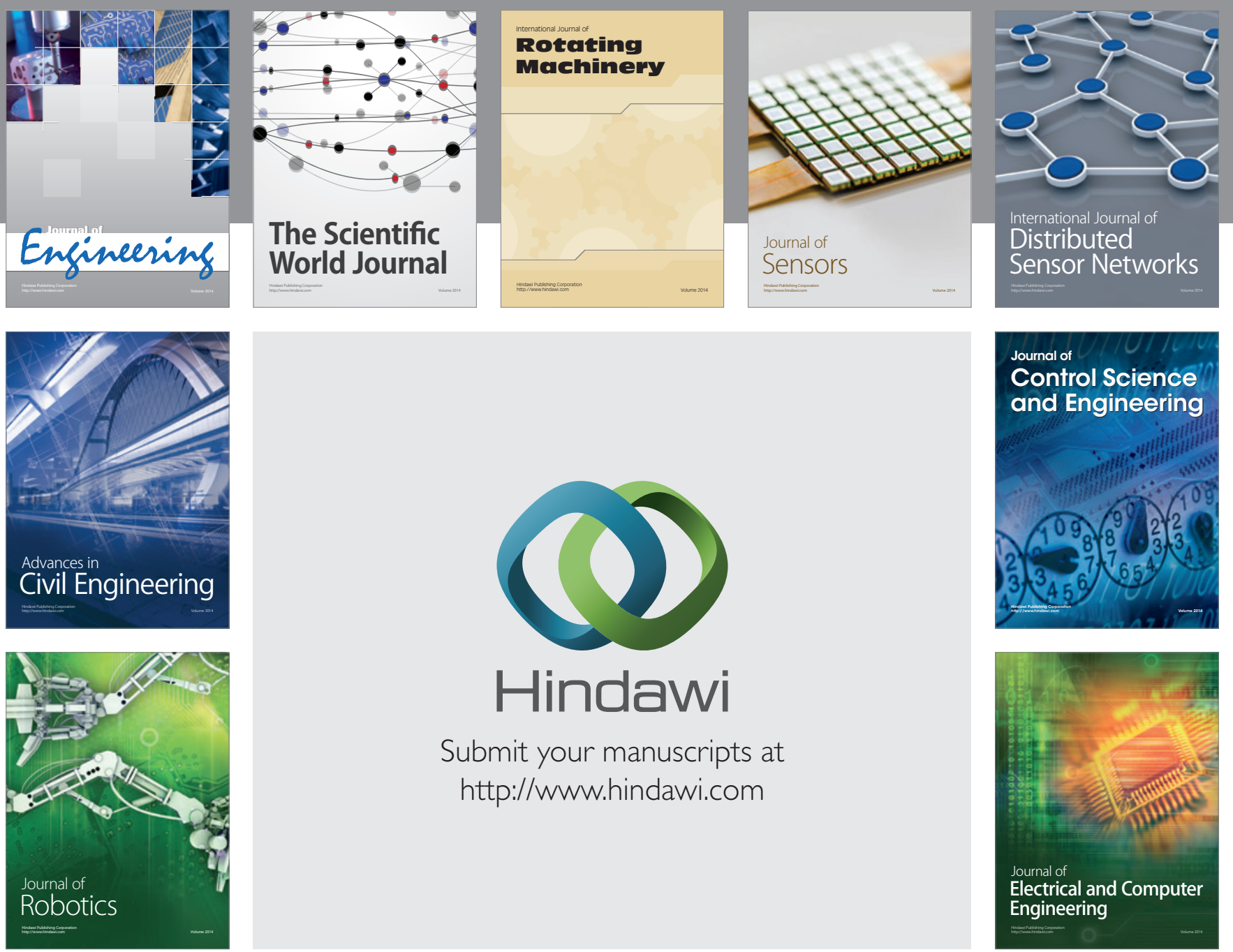

Submit your manuscripts at

http://www.hindawi.com
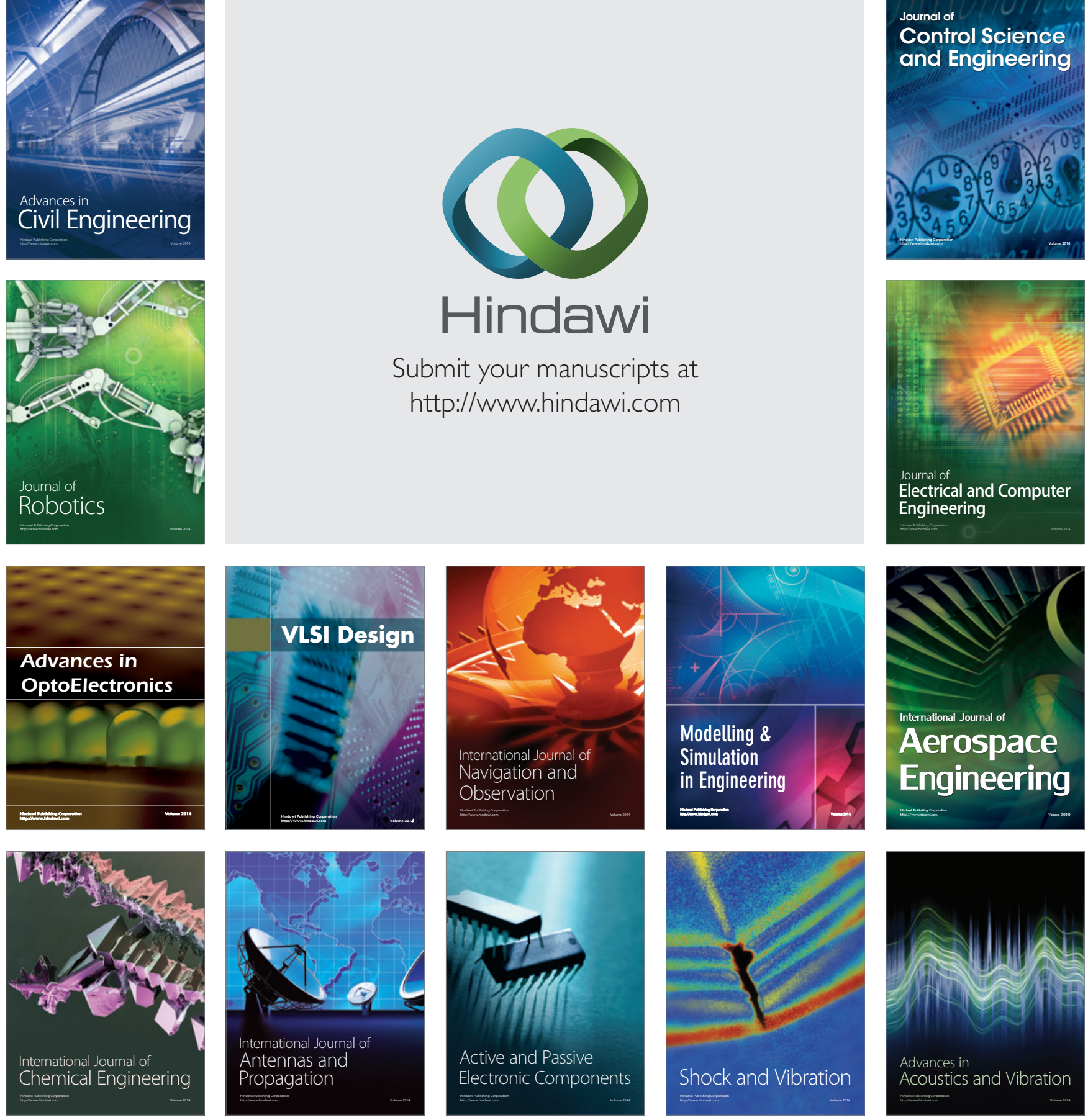